\title{
Le travail de la souffrance
}

Parcours biographique du cultivateur sénoufo (Côte d'Ivoire)

The work of suffering: The biographical itinerary of Senufo farmers

\section{Marianne Lemaire}

\section{CpenEdition}

\section{Journals}

Édition électronique

URL : http://journals.openedition.org/span/686

DOI : 10.4000/span.686

ISSN : 2268-1558

Éditeur

École pratique des hautes études. Sciences humaines

Édition imprimée

Date de publication : 1 juin 2005

Pagination : 71-90

ISSN : 0294-7080

Référence électronique

Marianne Lemaire, "Le travail de la souffrance », Systèmes de pensée en Afrique noire [En ligne],

17 | 2005, mis en ligne le 05 juin 2013, consulté le 01 mai 2019. URL : http://journals.openedition.org/ span/686 ; DOI : 10.4000/span.686 


\section{Le travail de la souffrance \\ Parcours biographique du cultivateur sénoufo (Côte d'Ivoire)}

\section{Marianne Lemaire}

Chargée de recherche au CNRS Centre d'études des mondes africains (CEMAf) CNRS/Université de Paris 1/

Université d'Aix-Marseille 1/EPHE

Les Sénoufo Tyebara, au nord de l'actuelle Côte d'Ivoire, ont très tôt retenu l'attention des voyageurs puis des administrateurs coloniaux pour leur ardeur dans le travail agricole. Mais chez ces observateurs, l'admiration le disputait toujours à la perplexité. En effet, ce que les Sénoufo célèbrent sous le nom de faliwi n'est pas exactement notre travail. Comme lui, le faliwi sénoufo incorpore une dimension de peine, de souffrance, mais c'est une souffrance valorisée pour elle-même, sans égard pour les œuvres qu'elle produit. De sorte que s'ils mettent dans la catégorie faliwi des activités que nous considérons comme du travail, ce n'est pas pour les mêmes raisons que nous. Et ils y incluent aussi des activités où il ne nous viendrait pas à l'esprit de voir du travail. C'est uniquement en raison de la souffrance qui accompagne leur réalisation qu'ils réunissent certaines de leurs activités sous la même catégorie faliwi, les élevant au rang de " travaux ». Parmi ces " travaux " figurent en bonne place différentes activités rituelles telles que celles accomplies par les novices lors de leur initiation au poro ${ }^{1}$, ou encore celles accomplies par ses proches et par les membres de son village lors des funérailles d'un défunt. Mais plus encore que ces différentes activités rituelles, le travail agricole fait figure de référence pour ces travaux sénoufo qui puisent leur valeur dans le douloureux effort qu'ils occasionnent plutôt que dans leur finalité productive.

\author{
${ }^{1}$ Deux articles d'Andras \\ Zempléni (1993 et \\ 1996) abordent la \\ question de la souf. \\ france initiatique et de \\ sa valorisation par les \\ Sénoufo.
}


On ne s'étonnera pas de ce qu'une souffrance à ce point valorisée soit attentivement évaluée en même temps que l'est la vaillance au travail de celui qui l'éprouve. Au cultivateur d'exception est en effet imputée une souffrance plus grande qu'à ses camarades. Mais cette souffrance en laquelle les Sénoufo voient un signe d'excellence se distingue moins par son intensité que par sa singularité. La souffrance du cultivateur d'exception est inattendue, et en augure une autre susceptible à son tour d'être appréhendée comme la preuve d'une nouvelle compétence. Ainsi, cet article se propose de retracer le douloureux parcours biographique que les Sénoufo réservent à leurs cultivateurs d'exception pour, à travers lui, tenter de préciser la nature de la souffrance qu'ils valorisent et situent à la source de l'excellence.

\section{La souffrance du champion de travail agricole}

La souffrance du travail agricole atteignait autrefois toute sa mesure au cours de concours de travail qui, sous leur forme la plus institutionnalisée, opposaient les cultivateurs de deux villages distincts dans un champ d'ignames et mobilisaient un public, des arbitres et des orchestres de xylophones. Que le travail agricole soit valorisé pour la souffrance qui l'accompagne et indépendamment de ses fruits n'apparaissait en effet jamais aussi clairement que dans le cadre de tels concours. Car la rivalité y accentuait une souffrance qui conduisait les cultivateurs à se retirer les uns à la suite des autres pour finalement céder la place aux seuls deux véritables rivaux dont ils s'étaient tout d'abord efforcés de suivre le rythme : les tegbanbele (pl. de tegban) ou champions de travail agricole de chacun des deux villages. "Trop de concurrence gâte le champ ", disent les Sénoufo : aussi vaillants que pouvaient être ces deux champions, ils ne pouvaient à eux seuls accomplir le travail de tout un collectif et laissaient un propriétaire de champ insatisfait face à une surface cultivée seulement en partie, et des buttes d'igname montées trop haut pour être utilisables. 
Mais la souffrance endurée par les deux rivaux n'était pas tout à fait la même que celle endurée par le commun des cultivateurs. En effet, le champion sur lequel ses camarades du même village et de la même classe d'âge comptent pour vaincre le champion du village adverse ne ressent aucune souffrance physique. De l'avis de tous, il est celui qui, alors que les autres cultivateurs s'épuisent à la tâche, ne sait pas qu'il en accomplit une : "Vous souffrez, lui ne sait pas que vous faites quelque chose "; il est encore celui qui dédaigne la douleur causée par une éventuelle blessure : "Même s'il se tape avec sa houe jusqu'à toucher l'os, il ne le considère pas, il cultive "; il est enfin celui qui ignore toute la journée durant les sensations de faim et de soif : «Il ne s'arrête pas pour manger, il n'a pas l'envie de nourriture ".

Des qualités qui se trouvent "sur son corps " et dont ses camarades sont dépourvus sont avancées pour justifier que le tegban soit comme épargné par la souffrance : ses os sont "durs" et son coeur, "bien assis ", ne "balance " pas. Ce n'est cependant pas «sur son corps » que se situent les ressources du tegban, mais bien au plus profond de luimême, "sur son cœur ». En effet, si le tegban ne souffre pas physiquement, c'est qu'il souffre d'une autre souffrance. "Son intérieur lui fait mal » : il est animé par une douloureuse rage de vaincre, un désir profond de " trouver le nom " à travers la réputation prestigieuse de meilleur cultivateur et de ne pas l'entacher par la honte et l'humiliation consécutives à l'insuccès. C'est une telle détermination qui manque au kanwolo, personnage qui s'oppose à celui du tegban sans pour autant se confondre avec celui du paresseux, car loin de refuser le travail, le kanwolo cultive avec soin et assiduité. Et si, malgré tout, les autres cultivateurs le dépassent parce qu'il ne cultive pas suffisamment vite, c'est parce que "son intérieur ne s'est pas arrêté sur cela » : parce qu'il n'est pas aussi douloureusement tendu vers la victoire que le tegban, il ne parvient pas à s'efforcer, redoute de voir souffrir ses os en cultivant trop énergiquement, de "sortir sous le soleil" ou de voir son cœur « se couper et tomber » : «Si tu répètes que cela te fait mal, tu ne peux pas t'efforcer comme les autres, tu vas rester derrière." 
${ }^{2}$ Les « chants de xylophones pour les cultivateurs à la houe » ont fait l'objet d'un précédent article dont je reprends ça et là quelques formulations (cf. Lemaire, 2000).
Ainsi, la souffrance morale du tegban, en étouffant sa souffrance physique, constitue sa meilleure assurance de le devenir ou de le rester : "Toute chose que tu veux faire, et que tu veux faire avec le cœur, elle va se faire. Si tu as peur d'elle à l'intérieur, elle ne se fera pas vraiment. " Aussi est-ce à l'élaboration progressive de cette souffrance particulière que travaillent les xylophonistes tout au long du concours. En effet, deux orchestres de xylophones sont postés au bord du champ, d'où chacun entonne des chants adressés tantôt à l'ensemble des cultivateurs et tantôt au tegban de son village $^{2}$. Qu'ils s'adressent à leur tegban, et les xylophonistes savent qu'ils ont à charge d'aiguillonner la douleur morale dont la possession lui a permis d'accéder au statut de tegban et dont l'exacerbation doit lui permettre de vaincre un adversaire lui-même tegban. Responsabilité qu'ils confessent clairement dans un de leurs chants, en soulignant que la " chose ronde tissée ", autrement dit la calebasse reliée à chacune des lamelles de bois que comporte leur instrument de musique, est mise en branle à seule fin de susciter la souffrance intérieure qui doit conduire leur tegban vers la victoire :

La chose ronde tissée nous a jetés dans cette souffrance.

Pour maintenir toujours vivace la souffrance morale de leur tegban, les musiciens ont recours à des chants qui portent sur le moment où plane l'incertitude concernant l'identité du vainqueur et où, pour cette raison, la tension atteint son paroxysme :

Ils cultivent vraiment, personne ne connaît le vainqueur parmi eux.

Incertitude et tension sont encore volontiers entretenues par l'évocation des divers accidents de parcours dont le tegban n'est jamais à l'abri et qui, s'ils survenaient, le voueraient indubitablement à l'échec :

Le manche de la houe du tegban ne doit pas se casser.

Mais les chants ne contribuent jamais autant au renforcement de la souffrance intérieure et, partant, au décuplement des forces du tegban tendu vers la victoire, que lorsqu'une jeune femme y est évoquée. Car cette image 
suscitée par les paroles des xylophones vient alors renforcer et magnifier deux autres figures féminines dont la présence est indispensable au bon déroulement du concours : les jeunes filles qui, munies d'une calebasse, offrent toute la journée durant de l'eau aux cultivateurs, et la statuette que le tegban est susceptible de remporter chez lui en guise de trophée. Sculptée au sommet d'une longue canne effilée à sa base, cette statuette, appelée « jeune fille des cultivateurs à la houe "(tefalapitya) $)^{3}$, représente effectivement une jeune femme tantôt debout, les bras le long du corps, tantôt assise sur un petit tabouret, les mains sur les genoux, tantôt claire, tantôt plus sombre, coiffée diversement et dotée d'attributs aussi divers qu'une houe, une jarre ou, plus rarement, un enfant. Or et cette statuette et les jeunes filles rappellent aux cultivateurs, et plus particulièrement au tegban, l'amie prénuptiale ou la future épouse pour laquelle ils cultivent d'ores et déjà ou celle dont leur ardeur au travail, attentivement évaluée par les aînés, pourrait leur faciliter l'obtention. Les xylophonistes, qui n'ignorent ni les sentiments ni les projets matrimoniaux du tegban qu'ils ont la responsabilité de rendre victorieux, s'appliquent à y conformer leur discours. Aussi leurs chants rappellent-ils volontiers ses connaissances féminines à la mémoire du tegban, à moins qu'ils n'évoquent une jeune personne connue de tous les villages à la ronde pour sa grande beauté et à laquelle un jeune tegban, plus que tout autre, est en droit d'aspirer :

Si quelqu'un a envie de Tyeden, qu'il parte dans le grand champ.

Que les xylophonistes soient véritablement résolus à lui frayer un chemin vers la victoire, et ils ne se satisfont pas de susciter une joute du tegban avec lui-même; ils s'appliquent également à amplifier la joute préexistante qui l'oppose à son principal adversaire. Là encore, les musiciens ne taisent pas le moyen auquel ils ont recours pour y parvenir : « jeter les deux tegbanbele l'un contre l'autre ", c'est à dire prêter des propos provocateurs à l'un pour aiguiser la souffrance et dans le même temps, la rage de vaincre de l'autre :

${ }^{3}$ Cette statuette n'a pas toujours revêtu la même forme au fil du temps. II semble en effet qu'à la mode de « la jeune fille des cultivateurs à la houe » ait succédé celle de "l'oiseau », un aigle dans certains villages, un vautour dans d'autres. 
Et c'est avec une grande satisfaction que les xylophonistes font finalement le constat de l'efficacité de leurs chants :

Nabege, si on te provoque pour te jeter contre les cultivateurs tu les cultives jusqu'à les gâter.

C'est ainsi une profonde souffrance qui est jugée indispensable à l'élévation d'un cultivateur au rang prestigieux de tegban et qui est entretenue par les xylophonistes tout au long du concours. Une profonde souffrance, mais une souffrance inattendue dans un contexte de travail agricole: une souffrance dont la dimension morale est continûment nourrie pour être en mesure d'étouffer sa dimension physique et de mener le tegban vers la victoire. Or cette surprenante souffrance avec laquelle les tegbanbele composent tout au long du concours, bien loin de prendre fin en même temps que lui, préfigure celle avec laquelle ils vont devoir composer tout au long de leur vie.

\section{Parcours biographique du tegban}

Que, en dépit de ses propres ressources et du soutien que lui ont apporté les xylophonistes de son village, le tegban échoue à vaincre son adversaire, et il se sent profondément humilié : son "intérieur" a été "noirci ", et son "derrière ", "arraché ». Il se voit également dépossédé de son prestige : s'il ne perd pas son titre au sein de son propre village, il perd la statuette tefalapitya ainsi que la considération et l'estime de son entourage, et ne les recouvre pas avant de remporter un prochain concours. Sa défaite le plonge dans un désespoir à la mesure de sa détermination à être le vainqueur, et encore augmenté par la déception des membres du village venus l'encourager : le mutisme des xylophones, les remontrances des anciens et les pleurs des jeunes femmes viennent encore théâtraliser l'échec ressenti douloureusement par le tegban. Dès lors, il peut arriver que la honte s'ajoute à l'épuisement pour entrainer, dès l'issue du combat, sa mort : le tegban ressent «l'envie de dormir, il se couche, il ne se réveille pas".

Vainqueur, le tegban est au contraire l'objet de nom- 
breuses attentions. De retour au village, il reçoit la meilleure part de nourriture et sa propre calebasse de bière. Les xylophonistes lui adressent des chants de louange sur lesquels seuls des champions de travail agricole aussi valeureux que lui sont autorisés à danser. Mais cet apaisement de la souffrance du champion de travail agricole est de courte durée. Soit qu'il perde son " nom ", et avec lui son prestige, soit au contraire qu'il le garde. Car les vainqueurs trop permanents de tous les concours auxquels ils participent s'exposent à une sanction inéluctable. Non pas parce que la figure du tegban irait à l'encontre de l'éthique égalitaire sénoufo. Tout occupé à accroître sa renommée et son prestige en participant au plus grand nombre de concours dans les villages alentours, le champion n'est pas assez souvent dans le sien pour prétendre y remplir des responsabilités. Si le tegban incarne une menace, c'est bien plutôt à l'endroit de la rivalité elle-même. L'ostracisme dont, comme on va le voir, il est bientôt l'objet évoque en effet celui qui, en Grèce ancienne, frappait les vainqueurs, et que Nietzsche interprète comme la volonté d'écarter ceux qui, par leur triomphe, mettaient en péril l'idée même de rivalité ${ }^{4}$.

A leurs plus grands champions de travail agricole, les Sénoufo Tyebara réservent un parcours douloureux tout au long duquel la statuette tefalapitya joue un rôle déterminant. Avec cette statuette, le tegban entretient en effet une relation étroite qui se révèle rapidement périlleuse. La tefalapitya n'est pourtant pas la propriété du tegban, mais celle de sa classe d'âge : ce sont les membres d'une classe d'âge en effet qui en ont fait ensemble la commande et payé le prix à un sculpteur, ou qui en ont hérité de leurs aînés n'en ayant plus l'usage. Ce sont encore les membres d'une classe d'âge qui, ensemble, choisissent de parer la statuette de boucles d'oreilles ou de colliers, conviennent de la couleur du pagne qu'elle portera, partent en quête des deux plumes, pareilles à celles arborées par le tegban, dont elle sera coiffée et qui, à la veille d'un concours, la lavent et la frottent avec de l'huile. Il faut donc attendre le début d'un concours pour que se précise, en même temps que sa relation très privilégiée avec le tegban, la véritable identité de la tefalapitya.

${ }^{4}$ Nietzsche, 1992, pp. 200-201. 
Pendant un concours, la statuette de chacun des deux villages est fichée en terre derrière son tegban et dans la dernière butte d'igname élevée par lui, un enfant étant chargé de la déplacer à mesure que le tegban progresse. Au moment où l'affrontement bat son plein, et où l'un des deux cultivateurs prend un avantage décisif sur son adversaire, sa tefalapitya se met, dit-on, à rire, à frapper des mains et à transpirer aussi abondamment que si on lui avait «versé du beurre de karité dessus ». Au même moment, son adversaire, en voie d'être battu, doit se rendre à l'évidence : le corps de sa statuette, comme le sien, blanchit. De fait, la tefalapitya est l'objet d'autres «soins " que ceux évoqués plus hauts, soins qui n'ont plus pour objectif de l'embellir, mais de l'animer, et qui ne sont plus du ressort des jeunes cultivateurs, mais des anciens et du tegban. Cette dimension magique de la statuette n'est pas sans présenter quelque danger pour celui dont elle est supposée renforcer les chances de victoire : par crainte des procédés et substances magiques dont la tefalapitya est le support, le tegban doit se garder de lui faire face, de piétiner son ombre ou d'en être recouvert, de la salir avec de la terre et plus encore d'en projeter dans le trou formé par la canne de la statuette après qu'elle a été déplacée. Qu'il néglige de respecter l'un de ces interdits, et il se voit " attrapé par le médicament » qui devait le rendre invincible, mais qui, finalement, le "cuit ": il se sent soudainement envahi par une fatigue et une mollesse insurmontables qui lui interdisent de cultiver et le contraignent à s'asseoir.

Là ne sont cependant pas les pires ennuis que la tefalapitya peut attirer au cultivateur d'exception. Elle est en effet au centre du parcours biographique semé d'embûches que partagent la plupart des plus grands champions. Parcours qui débute presque toujours ainsi : le cultivateur d'exception est aux prises avec des rêves étranges. Tel a été le cas de Pana Silué, ancien tegban du village de Zemongokaha, aujourd'hui âgé d'environ quatre-vingt dix ans. Pendant longtemps, Pana n'a pas dormi sans se réveiller avec, à l'esprit, le souvenir d'un rêve étrange : une jeune femme pénétrant dans sa chambre, s'adossant contre le mur puis venant se coucher près de lui. De ce rêve troublant, nombreux sont 
les devins qui lui ont donné cette première interprétation : sans doute Pana avait-il, "derrière lui ", une "personne d'un autre accouchement " (seelasyon) - une personne qui, parce qu'elle avait croisé Pana dans une vie antérieure et que celui-ci lui avait causé du tort, s'échappait de temps à autre du pays des morts pour à son tour semer le désordre dans sa vie. Car rêver chaque nuit d'une même femme n'affecte pas seulement le sommeil, mais aussi le temps de veille du rêveur : sans qu'il comprenne pourquoi, il entre en conflit avec tous les membres de son entourage, et tout particulièrement avec ses membres féminins. Qu'il ne soit pas encore marié, et il voit toutes les femmes le refuser; qu'il soit marié, et il constate que son épouse, outre qu'elle est en constante opposition avec lui, n'enfante pas. Les devins consultés par Pana avaient pu déterminer le clan d'appartenance de sa seelasyom : à une personne du même clan Yeo qu'elle, il avait alors remis le nombre d'ignames, la quantité de riz, de maïs et de condiments que les devins avaient estimé nécessaire pour qu'elle le « quitte ». Comme rien n'y faisait, Pana a consulté de nouveaux devins qui lui ont proposé une autre interprétation de son rêve : plutôt qu'une seelasyson, la femme qui le poursuivait en rêve n'était autre que la tefalapitya, la statuette dont il était alors, en tant que tegban, le détenteur. Ils ont ajouté cette précision : c'est parce que l'ardeur au travail de Pana a plu à la tefalapitya que celle-ci le « colle " aujourd'hui avec autant d'insistance.

Plus jeune que Pana d'une dizaine d'années, mais ancien champion de travail agricole comme lui, Zananyene Soro, du village de Dihi, a également commencé par rêver chaque nuit d'une même jeune femme. Les devins lui ont plus promptement qu'à Pana exposé de quoi il retournait : "Les devins me l'ont dit : ils m'ont dit que le morceau de bois avec lequel je cultivais, c'était ce qui s'était collé à moi. " L'époque des premiers rêves de Zananyene a coïncidé avec le moment précis où sa classe d'âge transmettait la tefalapitya à ses cadets. Il l'explique ainsi : tout d'abord séduite par son ardeur au travail, la tefalapitya a par la suite si activement participé à accroître sa réputation de tegban qu'elle n'a pu se résoudre à se séparer de lui : "Dans le champ, on aurait 
dit que je ne cultivais pas tout seul. Quand nous avons eu fini de cultiver avec elle, de la manière dont elle m'avait aidé dans le travail agricole, elle n'a pas accepté de me quitter. » Fort de cette certitude que la tefalapitya s'était effectivement collée à lui "sur le lieu du travail agricole ", Zananyene a tout mis en œuvre pour qu'elle cesse de l'importuner. Son premier geste a été de la dessiner : apercevant sa propre silhouette tracée au kaolin sur le mur de la chambre de Zananyene, elle ne devait plus pouvoir y pénétrer. Dans le même objectif, Zananyene enfumait quotidiennement sa maison en y faisant brûler les mêmes herbes qu'il brûlait autrefois pour que la tefalapitya lui apporte son soutien lors des concours entre cultivateurs. Mais tous ses efforts pour éloigner de lui la tefalapitya se sont soldés par un succès aussi variable que temporaire. Elle est toujours revenue, manifestant son mécontentement à l'égard de son "ami " à travers des gestes meurtriers : deux des trois épouses de Zananyene sont mortes après avoir donné naissance à des enfants qui n'ont eux-mêmes pas survécu. Aussi Zananyene a-t-il fini par se plier à toutes les exigences de la tefalapitya. Il a en premier lieu fait sculpter une statuette la représentant, puis quelques temps plus tard, une seconde statuette masculine représentant l'" ami " qu'elle souhaitait avoir à ses côtés. Deux fois l'an il sacrifie une poule au plumage roux à la statuette féminine sculptée à l'image de la tefalapitya avec laquelle il cultivait, et un coq blanc à la statuette masculine. Il prend alors soin de verser le sang à leurs pieds plutôt que sur leur tête, ainsi qu'elles le lui ont réclamé par le biais des devins, et présente à chacune un plat de nourriture. Toujours à leur demande, Zananyene a ensuite et tour à tour acquis pour les déposer près d'eux tous les objets qui constituent l'équipement du devin : le bracelet en forme de python, la calebasse-hochet, les icônes divinatoires et le panier des jumeaux. Zananyene s'est enfin résolu à respecter le jour d'interdit de travail que la tefalapitya lui imposait - le jour yalamusa, au cours duquel il s'abstient non seulement de cultiver, mais aussi de se promener et de parler avec quiconque.

Il est cependant un point déterminant sur lequel 
Zananyene ne lui a jamais cédé. Comme elle lui demandait depuis toujours de rejoindre le corps des initiés au sandogi, l'ancien champion de travail agricole ne lui a jamais répondu en d'autres termes que ceux-ci : «Je préfère que tu me tues." Le terme sandogi désigne l'institution initiatique dont la puissance du même nom est au centre et qui, contrairement au poro, recrute presque exclusivement des femmes, et les recrute par voie d'élection : un ancêtre utérin ou, plus fréquemment, un génie, « choisit » la petite fille ou la femme adulte que, parce que celle-ci lui a plu ou, au contraire, déplu, il souhaite voir subir l'initiation au sandogi. Le refus catégorique de Zananyene d'intégrer l'institution initiatique du sandogi se comprend sans mal : le statut d'initié au sandogi (sandowi, pl. sandobele) va de pair avec l'obligation d'observer quotidiennement un nombre considérable de prescriptions et proscriptions. Parmi ces dernières figure un interdit plus redoutable et redouté que tous les autres : un interdit de travail agricole. Il est fort probable en effet que le premier mouvement d'un génie soit d'exiger de celui qu'il a élu de ne plus " se salir avec la terre » et, partant, de « laisser la souffrance du travail agricole ». Certes, tous les génies n'ont pas les mêmes exigences, et quelques rares initiés au sandogi sont autorisés à cultiver sans aucune restriction, si ce n'est celle du jour hebdomadaire de repos que son yigevswi, son double protecteur, impose à chaque Sénoufo. D'autres, plus nombreux, sont autorisés à cultiver dans les rizières, sinon dans la terre "sèche "; d'autres encore sont autorisés à cultiver avec tel outil, sinon avec tel autre. Mais plus nombreux encore, amplement majoritaires, sont les initiés au sandogi qui ne sont autorisés à cultiver à aucun moment, sur aucune terre, avec aucun outil. Aux oreilles des cultivateurs que sont les Sénoufo, une telle sommation du génie sonne comme une condamnation sans appel. À celles du champion de travail agricole, elle sonne sans aucun doute plus amèrement encore. Car c'est alors celui qui excellait dans le travail agricole qui doit, en raison même de son excellence, y renoncer. C'est un tel renoncement que Zananyene a, de justesse, pu éviter. Pana, comme la grande majorité des champions de travail agricole, n'a pas eu la même chance. 
Celle qui avait finalement été identifiée par les devins comme la tefalapitya perturbait toujours son sommeil. Alors, Pana a continué de satisfaire ses requêtes successives et notamment passé commande auprès d'un sculpteur de ses amis d'une statuette la représentant, et la représentant telle qu'elle voulait être représentée : une jeune femme sur le dos d'un cheval. Pendant un temps, la jeune démone ne s'est plus manifestée à lui au cours de son sommeil. Mais elle est bientôt revenue, plus dure et plus tyrannique que jamais : d'ores et déjà responsable des désagréments que ses rêves causaient à Pana, elle s'est alors rendue responsable d'infortunes bien plus sérieuses : après qu'un serpent l'a mordu, Pana a perdu deux doigts de son pied gauche et est resté alité toute une année au cours de laquelle il a chaque jour pensé mourir le lendemain. C'est alors que les devins lui ont précisé l'exigence ultime du génie masqué par la tefalapitya : il devait cesser de cultiver et prendre le statut de sandowi. Pana s'y est très longtemps refusé. Encore très jeune, il était peu disposé à mener la vie contraignante qui est celle de l'initié au sandogi. Il regrette néanmoins son choix : eût-il plus rapidement accepté de " porter " le sandogi et de cesser de cultiver ainsi que le génie le lui demandait, et il n'aurait pas perdu consécutivement trois épouses et huit enfants. Il a fallu un dernier événement pour que Pana se rende à l'évidence : alors qu'il cultivait, il a vu un petit personnage rouge à ses côtés. Quand il s'est aperçu que lui seul le voyait, il a déposé sa houe et ne l'a plus jamais reprise.

Comme l'avalanche de ses infortunes a cessé dès l'instant où il n'a plus cultivé, Pana sait aujourd'hui que les devins disaient vrai : un génie abrité par la tefalapitya l'a choisi "sur le lieu du travail agricole ", et en raison de son ardeur au travail agricole. Mais il sait aussi, comme Zananyene, qu'il doit à ce jeune génie femelle d'avoir dans un premier temps conforté son statut de tegban. Séduite par son ardeur au travail, elle lui a tout d'abord apporté son soutien lors des concours qui l'opposaient à d'autres cultivateurs d'exception : «Au début, tu ne le sais pas. Mais si tu commences à cultiver, tu n'es jamais rassasié de travail, tu as envie de cultiver, tu ne te fatigues pas. C'est qu'elle cultive 
avec toi. " Jusqu'au jour où le même génie qui avait permis à Pana d'atteindre le sommet de sa gloire a exigé qu'il cesse de cultiver : "Le jeune génie femelle, c'est lui qui m'a dit de m'asseoir, d'arrêter le travail agricole. Elle n'a plus voulu que je me salisse avec la terre, elle n'a plus voulu que la saleté reste près d'elle. »

Or "laisser la souffrance du travail agricole", ainsi que votre instance électrice vous le demande, revient à en expérimenter une autre. Au cours de la même consultation divinatoire où le devin vous apprend qu'un génie souhaite vous voir renoncer à votre travail et vous " asseoir ", il vous apprend également que ce génie souhaite vous voir accomplir un autre travail : le sien. "Ton génie te dit de t'asseoir pour faire son travail ", "il te dit de prendre ses bagages »: à l'aide de ces différentes formulations, le devin signifie à l'ancien champion de travail agricole que son génie souhaite le voir rejoindre le corps des initiés au sandogi et transmettre sa parole à travers la divination.

\section{La souffrance du devin}

Les Sénoufo Tyebara pratiquent une technique divinatoire combinant des jets d'icônes et des frappements de main. Les consultations se déroulent dans l'intimité de la maison miniature dont dispose tout devin pour y abriter les différents objets composant ses « bagages » d'initié. Lorsqu'il y pénètre, le client a pour premier geste de déposer le prix de la consultation dans un petit panier prévu à cet effet. Puis il s'assoit aux côtés du devin, dans le sens opposé. Les premiers mots du devin sont pour inviter les génies dont il s'apprête à transmettre les paroles à le rejoindre. Quand il a le sentiment que ces génies ont répondu à son appel et que, installés auprès de lui, ils sont attentifs et disposés à répondre à ses questions, il procède à un premier lancer de ses icônes divinatoires. Leur disposition sur le sol doit lui permettre de déterminer, approximativement au moins, le motif de la venue de son client. Après avoir jeté ses icônes autant de fois qu'il l'a jugé nécessaire, le devin saisit la main de son client. Il adresse alors à ses génies une série de 
questions, auxquelles le mouvement que tendent à prendre les mains jointes des deux partenaires donnera la réponse : positive si elles viennent claquer sur la cuisse du devin, négative si elles se balancent au-dessus d'elle. Au cours de cette étape centrale de la consultation, les réponses que lui apportent ses génies permettent au devin de préciser les contours de l'infortune de son client, d'identifier l'instance ou l'individu qui en est à la source et enfin de spécifier son mode approprié de résolution. C'est seulement dans la troisième phase de la consultation divinatoire que le client est invité à s'exprimer : ses questions peuvent éventuellement nécessiter la réitération des lancers d'icônes divinatoires ou des frappements de main.

Une fois engagé dans la divination, notre ancien champion de travail agricole souffre d'une souffrance morale qui lui est familière pour l'avoir tenaillé lors des concours de travail agricole mais aussi, plus tard, pendant son initiation au sandogi. En effet, tous les devins appartiennent au corps des sandobele et la plupart d'entre eux ont eu à subir une initiation moralement éprouvante. Composée de deux étapes principales, celle-ci s'étend le plus souvent sur un an et demi. Il ne semble pas qu'au cours de ce laps de temps, le novice ait à traverser des épreuves physiques aussi pénibles que celles des novices du poro. Plus que son corps, c'est en effet son cœur que les épreuves initiatiques du sandogi mettent à rude épreuve. Car au contraire des novices de l'initiation au poro, le futur sandowi accomplit seul un travail dont il n'a jamais la certitude qu'il lui revient, à lui, de l'accomplir. Il n'est jamais exclu en effet qu'un malveillant qui souhaite la mort d'une personne force magiquement les devins à lui dire de "porter " le sandogi. Elle le "porte ", mais parce que tel n'était pas son destin, elle meurt. C'est pourquoi les proches du novice l'observent attentivement tout le temps que dure son initiation, et tout particulièrement au terme de la première semaine d'initiation au cours de laquelle le novice "attache" le sandogi: si les devins ont réellement dit la vérité en affirmant qu'il était élu, il doit avoir pris du poids. Quoi qu'il en soit, il n'est plus temps de revenir en arrière : le sandogi est d'ores et déjà « soulevé ". La dernière étape de 
l'initiation, intitulée "habiller le sandogi " comprend elle aussi son moment de vérité. De même que «attacher le sandogi ", elle s'étend sur une semaine; mais contrairement à elle, elle a lieu au cours de la saison pluvieuse. Le novice est, avant toute chose, rasé une dernière fois. Il est ensuite conduit en brousse où il lui est ordonné de prendre le trriwi, un objet connu des seuls initiés. Le temps qu'il lui faut pour trouver l'objet dissimulé est inversement proportionnel au temps qu'il lui reste à vivre. Il se peut aussi qu'il le cherche en vain : le sandogi qu'il a pourtant d'ores et déjà attaché n'était donc en réalité pas le sien, et il le tuera sans doute prochainement. Il reste que les sandobele ne rentrent jamais de brousse sans un chant à la bouche qui affirme avec conviction que "notre enfant va vieillir, notre enfant va vivre ». Ainsi, l'initiation au sandogi se présente comme une construction laborieuse de la certitude que, puisque le sandogi que l'on « dépose " sur le novice est bien celui qu'il devait porter, il ne va pas le tuer 5 .

Et encore le doute poursuit-il le sandowi bien au-delà de son initiation : tous les membres de son entourage ne verront pas d'un bon oeil sa dispense de travail agricole, et il est même probable que quelques-uns l'interprètent comme de la paresse. C'est d'ailleurs pour cette raison que les sandobele, quitte à " porter " un sandogi, préfèrent " porter " celui que l'on trouve «sur le lieu du travail agricole». Car c'est là la manière la plus noble de le trouver, ou tout au moins celle qui éveille le moins la suspicion, ainsi que l'explique Pana : "Ceux qui le trouvent ailleurs que dans le travail agricole, nous les appelons paresseux. Car si quelqu'un ne veut pas cultiver, il ment et dit qu'un génie s'est collé à lui. " Et c'est encore pour cette raison que les sandobele préfèrent que le «travail » de leur génie ne consiste pas seulement à respecter une suite d'interdits, mais comprenne également une prescription, celle de pratiquer la divination. Car au sandowi est alors donné la possibilité d'exercer un nouveau travail appréhendé par tous comme tel. Or si tous les sandobele ne sont pas devins, tous ceux qui le sont devenus en tant que champions de travail agricole sont sommés par leur génie de transmettre sa parole.

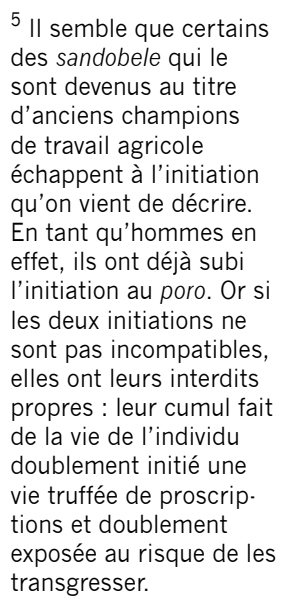


Mais une fois devin, l'incertitude continue de tenailler l'ancien champion de travail agricole. Car l'une des plus grandes souffrances du devin est d'ignorer si, en transmettant les paroles de ses génies, il dit la vérité. Simple médiateur, le devin dépend de ses génies pour identifier correctement l'infortune de son client, pour lui en proposer une interprétation juste et pour prescrire un mode de résolution approprié. Une telle incertitude quant à la validité des réponses que ses génies donnent à ses questions est à la source d'une profonde souffrance morale. Non qu'il puisse être jugé coupable de "mensonges" qui ne sont pas les siens, mais qu'il puisse pâtir des "mensonges " de ses génies. Car si les devins ne jouissent pas tous de la même réputation, les devins réputés ne sont jamais assurés de pouvoir maintenir leur renommée : "Ne me faites pas honte, n'abaissez pas ma tête avec vos mensonges. "Il n'est pas question d'autre chose que de cette douloureuse incertitude dans cette prière qui, au début d'une nouvelle saison pluvieuse, accompagnait un don sacrificiel de Pana à son génie :

Tu me dis de donner un mouton, génie.

Au moment de ma jeunesse, tu m'avais choisi.

Je te dis de prendre la parole et de la donner aux gens de Sandope, qu'ils la prennent et la donnent aux gens de Gbe'elっ's, qu'ils la prennent et la donnent aux gens de Kondo, qu'ils la prennent et la donnent à mon père.

Tu dis que je n'ai pas accepté de prendre ton travail, que c'est pourquoi tu as empêché ma réussite.

Je dis que je te demande pardon, le jour comme la nuit.

Je te dis de trouver quelque chose à me donner.

Donne-moi la santé, donne-moi le plat et la sauce, donne-moi un lieu où manger.

Le problème auquel je songe au fond de moi, mets-le dans ma main.

Ainsi que tu m'as dit de m'asseoir, je me suis arrêté pour m'asseoir.

Laisse les gens se regrouper, fais que le monde entier connaisse mon nom.

Que l'on dise : "Si tu vas chez Pana et qu'il ramasse du sable pour te le donner, cela va faire de ta vie une réussite. »

$\mathrm{Si}$ tu entends qu'un génie a surpris une personne, il est son yigevowi.

Si un homme ou une femme dit: "Je vais chez Pana pour faire du mensonge." 
Génie, si tu es vrai, laisse que je le sache, laisse que je connaisse cette personne.

C'est ta bouche qui m'a dit de m'asseoir, et je dis que j'ai accepté de m'asseoir.

Etre assis ne parle pas, c'est ce qui est derrière qui parle.

$\mathrm{Tu}$ avais dit que je te plaisais et tu m'avais choisi.

Je te dis de laisser les gens venir vers moi à tout moment.

Que les femmes viennent, que les hommes viennent.

Je suis assis, je ne cultive pas, et je te dis de laisser les gens me connaître.

Si ta personne doit mourir sans un [bon] nom, ce n'est pas la peine.

Les bagages que tu as chargés sur moi, ce ne sont pas les miens, ils restent les tiens.

C'est toi qui vas arracher la tête de ta faiblesse.

Si une personne ne me connaît pas, tu vas entendre de sa part : «Suis-je du genre du génie de Pana, un génie de beaucoup de problèmes, il dit de donner une poule, un bœuf, un mouton, il les tue et les mange parce qu'il a envie de manger de la viande, mais il ne fait rien de bon. "

Plutôt qu'elle ne m'insulte, c'est toi que la personne insulte.

Si une femme se lève, si un homme se lève en disant " la tête de Pana ", qu'elle se perde.

Si une personne dit que je ne lui plais pas, permets que je la dépasse en marchant dans la fraîcheur de son eau.

Si une personne éternue, elle ne vaut pas un génie, et je dis que je t'ai donné ma tête.

Permets que les femmes le sachent, permets que les hommes le sachent.

Voici le mouton de la rentrée de la saison pluvieuse.

Mais comme le champion de travail agricole qu'il était autrefois, le devin souffre également d'une souffrance inattendue. Car pratiquer la divination est un véritable "travail ", et un travail comparable au travail agricole. De sorte que contre toute attente, les Sénoufo imputent au devin une souffrance physique à la mesure de sa souffrance morale et semblable à celle du cultivateur. Tout comme le cultivateur abaissé sur son sillon, le devin a «mal au corps ». Tout comme lui, il travaille "avec les mains ", et il les « chauffe ». Tout comme lui, il « frappe ", et frappe non pas la terre, mais son propre corps : là où il frappe, le sang "se coagule" de sorte que la peau devient noire et dure ${ }^{6}$. «Frapper avec la main » est d'ailleurs si douloureux que ses génies ont récemment permis au vieux Pana d'y renoncer.

${ }^{6}$ Les Sénoufo aiment à rappeler que, comme le cultivateur également, le devin manipule, en guise d'icône divinatoire déterminante entre toutes, une houe minia. turisée. 
"Taper avec la main " serait cependant moins éprouvant si le devin sénoufo n'était pas incité à frapper si fort. Quand, en effet, un simple frappement de main du devin contre sa cuisse indique une réponse positive du génie, un puissant et retentissant frappement de main du devin contre sa cuisse indique une réponse sans nul doute positive du génie. Du devin réputé pour la constante véracité de ses dires, les Sénoufo diront qu'il devine " avec force ». Tout se passe ainsi comme si l'effort et la souffrance investis par le devin dans ses gestes investissaient à leur tour ses paroles d'authenticité, et soulageaient du même coup sa souffrance morale.

Le parcours biographique du champion de travail agricole est celui d'un être d'exception qui n'en est jamais quitte avec la souffrance. En effet, les Sénoufo imputent une souffrance immense à celui qui excelle dans le champ du travail agricole avant d'exceller dans celui de la divination. Mais cette souffrance, qu'elle conditionne l'excellence du cultivateur ou celle du devin, est toujours surprenante. Car contre toute attente, c'est une souffrance plus morale que physique qui est imputée au tegban, et une souffrance aussi physique que morale qui est imputé au devin. Tout se passe en effet comme si les Sénoufo complétaient la souffrance de leurs êtres d'exception en la nourrissant de la dimension tantôt morale et tantôt physique qui lui fait le plus défaut. Avant que de la situer à la source de l'excellence, les Sénoufo façonnent une souffrance plénière.

Prenons garde cependant que cette souffrance plénière n'est pas une souffrance augmentée. Les Sénoufo, certes, valorisent la souffrance, mais ils la valorisent en tant qu'elle comprend en elle-même son propre soulagement. Et s'ils lui apportent un complément, ce n'est en aucun cas dans le but de l'intensifier, mais dans celui de l'édifier telle qu'ils la valorisent. C'est ainsi que la souffrance morale du champion de travail agricole ne se surajoute pas à sa souffrance physique, pas plus que la souffrance physique du devin ne se surajoute à sa souffrance morale. Bien au contraire, la 
souffrance morale du champion soulage sa souffrance physique, quand la souffrance physique du devin atténue sa souffrance morale. De tant souffrir au fond de lui-même, le tegban en oublie de souffrir "sur son corps ». A l'inverse, de souffrir physiquement donne au devin l'assurance de transmettre la vérité à ses clients, et adoucit son tourment.

C'est ainsi bel et bien une souffrance entière que les Sénoufo situent à la source de l'excellence du champion de travail agricole et du devin, mais c'est une souffrance qui, parce qu'elle est entière, permet à celui qui l'expérimente d'y trouver les forces de la surmonter. Un peu comme Saint Jean de la Croix pour qui, selon Alain Cugno ${ }^{7}$, « la mortification n'est pas un moyen pour augmenter en [s]oi la souffrance, mais au contraire pour la diminuer, en faisant sentir à [s]on corps la douleur qui est tenable, par opposition à la souffrance qui est intenable ». "De celui qui mord son poing parce qu'il souffre trop ", poursuit Alain Cugno, « il serait assez inexact de prétendre qu'il cherche à augmenter sa souffrance. "Il n'en va pas autrement pour les Sénoufo Tyebara, qui imputent à leurs êtres d'exception, dans les champs successifs où ils excellent - le travail agricole puis la divination - une douleur tenable, construite de toutes pièces, à même de soulager la souffrance véritable, celle, physique, du travail agricole et celle, morale, du travail divinatoire. De sorte que si les Sénoufo ne conçoivent pas d'excellence sans souffrance, ils ne conçoivent pas non plus de souffrance sans, en son sein précisément, le moyen de la surmonter. 


\section{Références bibliographiques}

Cugno, A.

1972 Saint Jean de la Croix, Paris, Fayard.

Lemaire, $\mathrm{M}$.

2000 "Chants de l'agôn, chants du labeur. Travail, musique et rivalité en pays sénoufo (Côte d'Ivoire) ", Journal des Africanistes 69 (2), pp. 35-65.

Nietzsche, F.

1992 [1870] "La joute chez Homère ", in La philosophie à l'époque tragique des Grecs, Paris, Gallimard, pp. 196-204.

Zempléni, A.

1993 "L'invisible et le dissimulé. Du statut religieux des entités initiatiques", Gradhiva 14, pp. 3-14.

1996 "Savoir taire. Du secret et de l'intrusion ethnologique dans la vie des autres ", Gradhiva 20, pp. 23-42. 